\title{
ON MATRIX APPROXIMATION ${ }^{1}$
}

\section{SHMUEL FRIEDLAND}

ABSTRACT. In this paper we give an algebraic characterization of the best approximants to a given matrix $A$ from a real line spanned by a matrix $B$. The distance $\|A-a B\|$ is taken to be the spectral norm of $A-a B$.

1. Introduction. Let $A$ and $B$ be $m \times n$-complex-valued nonzero matrices. Assume that $A \neq \alpha B$ for any real $\alpha$. We are looking for

$$
\min _{\alpha \in \mathbb{R}}\|A-\alpha B\|=d .
$$

Here $\|A\|$ is the spectral norm of $A$, i.e., the square root of the greatest eigenvalue of $A^{*} A$. The special case where $A$ is a real $n \times n$-matrix and $B$ is the identity matrix $I$ is interesting in the theory of partial differential equations [1]. It is well known that the set of all real $\beta$, such that

$$
\|A-\beta B\|=d
$$

is a compact convex set. That is, this set is of the form

$$
\alpha_{1} \leq \beta \leq a_{2} \quad\left(a_{1} \leq a_{2}\right)
$$

In what follows, we characterize $\alpha_{1}$ and $\alpha_{2}$ as solutions of certain polynomial equations.

2. The main result. Let

$$
s(\lambda, \alpha)=\lambda^{n}+s_{1}(\alpha) \lambda^{n-1}+\cdots+s_{n}(\alpha)
$$

be the characteristic polynomial of the matrix $\left(A^{*}-\alpha B^{*}\right)(A-\alpha B)$. Thus we consider $S(\lambda, \alpha)$ as a polynomial in $\lambda$ over the polynomial ring $P[\alpha]$. Let $D_{1}(\lambda, \alpha)$ be the greatest common divisor of $S(\lambda, \alpha)$ and $\partial S(\lambda, \alpha) / \partial \lambda$ over the field $F[\alpha]$ of all rational functions in $\alpha$. However, as the leading coefficient of $S(\lambda, \alpha)$ and $\partial S(\lambda, \alpha) / \partial \lambda$ do not depend on $\alpha$, it is easy to show that $D_{1}(\lambda, \alpha)$ can be chosen as a polynomial over $P[\alpha]$, whose leading

Received by the editors May 29, 1974. $65 \mathrm{~F} 35$.

AMS (MOS) subject classifications (1970). Primary 15A60, 15A18; Secondary

Key words and phrases. Matrix approximation, spectral norm, polynomial equations, the greatest common divisor, discriminant.

1 This paper partially supported by NSF Grant 28876 and Navy Contract N0001467-A-0112-0015. 
coefficient does not depend on $\alpha$. By $R(\lambda, \alpha)$, we denote the reduced characteristic polynomial

$$
R(\lambda, \alpha)=S(\lambda, \alpha) / D_{1}(\lambda, \alpha)
$$

Again, $R(\lambda, \alpha)$ is a polynomial over $P[\alpha]$. Thus if $s\left(\lambda_{0}, \alpha_{0}\right)=0$, then $R\left(\lambda_{0}, \alpha_{0}\right)=0$. By the definition of $R(\lambda, \alpha)$, the discriminant $\rho(\alpha)$ of $R(\lambda, \alpha)$, i.e., the resultant of the polynomials $R(\lambda, \alpha)$ and $\partial R(\lambda, \alpha) / \partial \lambda$, is a nonzero element in $P[\alpha]$. Denote by $D_{2}(\lambda)$ the greatest common divisor of $R(\lambda, \alpha)$ and $R\left(\lambda, \alpha_{0}\right)$ over $F[\alpha]$. It means that the roots of $D_{2}(\lambda)$ are all the roots of $R(\lambda, \alpha)$ which do not depend on $\alpha$. By $Q(\lambda, \alpha)$, we denote the polynomial

$$
Q(\lambda, \alpha)=R(\lambda, \alpha) / D_{2}(\lambda)
$$

over $P[\alpha]$.

Theorem. Let $A$ and $B$ be $m \times n$-complex-valued nonzero matrices. As-

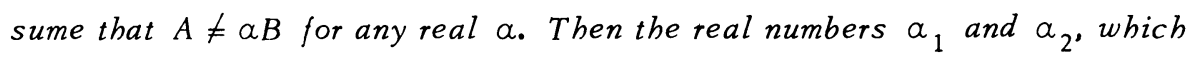
are defined by the conditions (2) and (3), satisfy either the equation

$$
\rho(\alpha)=0
$$

or the system

$$
Q(\lambda, \alpha)=\partial Q(\lambda, \alpha) / \partial \alpha=0 .
$$

The equation (7) and the system (8) have only a finite number of solutions.

3. Proof. Assume first that $\alpha_{1}<\alpha_{2}$. So $\|A-\beta B\|=d$ for $\alpha_{1} \leq \beta \leq \alpha_{2}$. This implies that $S\left(d^{2}, \alpha\right)=R\left(d^{2}, \alpha\right)=0$ identically. We claim that, for $\alpha=a_{j}, j=1,2, d^{2}$ is at least a double root of $R(\lambda, \alpha)$. Suppose that $d^{2}$ is a simple root for $\alpha=\alpha_{j}$. From (2), we deduce that all other roots of $R\left(\lambda, \alpha_{j}\right)$ are strictly less than $d^{2}$. Thus if $\left|\alpha-\alpha_{j}\right|<\epsilon, d^{2}$ is the greatest root of $R(\lambda, \alpha)$ for an appropriate positive $\epsilon$. So $\|A-\alpha B\|=d$ for that $\alpha$. This contradicts the definition of $\alpha_{1}$ and $\alpha_{2}$. Thus in the case that $\alpha_{1}<\alpha_{2}, \alpha_{j}$ has to satisfy (7) for $j=1,2$. Since $\rho(\alpha)$ is a nontrivial polynomial, there exist only finite numbers of solutions. (It may happen that $\rho(\alpha) \equiv$ Const, which means that $\alpha_{1}=\alpha_{2}$.) Assume now that $\alpha_{1}=\alpha_{2}=\alpha^{*}$. If $d^{2}$ is at least a double root of $R\left(\lambda, \alpha^{*}\right)$, then $\alpha^{*}$ has to satisfy (7). Suppose now that $d^{2}$ is a simple root of $R\left(\lambda, \alpha^{*}\right)$. Thus for $\left|\alpha-\alpha^{*}\right|<\epsilon$, there exists an analytic function $\lambda(\alpha)$ which is defined by the equation

$$
R(\lambda, \alpha)=0
$$

and $\lambda\left(a^{*}\right)=d^{2}$. This is because $\partial R(\lambda, \alpha) / \partial \lambda \neq 0$ on this neighborhood of $\left(d^{2}, a^{*}\right)$. We restrict ourselves to this neighborhood. Obviously, $\lambda(\alpha)$ is not 
a constant function. Otherwise, $\|A-\alpha B\|=d$, which contradicts the assumption that $\alpha_{1}=\alpha_{2}=\alpha^{*}$. So $\lambda(\alpha)$ is the greatest root of $Q(\lambda, \alpha)=0$. Since $\lambda(\alpha)>d^{2}$ for $\alpha \neq \alpha^{*}$, we conclude that $d \lambda\left(\alpha^{*}\right) / d \alpha=0$. This proves that $\alpha^{*}$ satisfies the system (8).

We show that (8) has only a finite number of solutions. First, note that all roots of $Q(\lambda, \alpha)$ are distinct except for a finite number of $\alpha$. This follows from the fact that $\rho(\alpha)$ is a nonzero polynomial. Thus if the system (8) has an infinite number of solutions, it would imply that the polynomials $Q(\lambda, \alpha)$ and $\partial Q(\lambda, \alpha) / \partial \alpha$ have a nontrivial common factor $D_{3}(\lambda, \alpha)$. But this means that $Q(\lambda, \alpha)$ has constant roots which do not depend on $\alpha$. This contradicts the definition of $Q$.

4. Open problem. Let $A$ and $B_{1}, \cdots, B_{k}$ be $m \times n$-complex-valued matrices. Assume that $B_{1}, \cdots, B_{k}$ are linearly independent on $\mathbf{R}$. Denote by $\mathcal{U}$ the real span of $B_{1}, \cdots, B_{k}$. An obvious extension of the problem discussed above is: Characterize the extreme points of the convex set of the best approximants $\mathcal{B}$. That is, $\mathcal{B} \subset \mathcal{U}$, and, for any $B \in \mathcal{U}$ and $C \in \mathcal{B}$,

$$
\|A-B\| \geq\|A-C\|=d \text {. }
$$

It seems that, from the case $k=1$, the extreme points of $B$ can be characterized by a set of certain polynomial equations.

Acknowledgment. The author wishes to thank P. R. Halmos for suggesting the problem, and $\mathrm{E}$. Deutsch for supplying the reference.

\section{REFERENCE}

1. I. A. Rus, Un principe du maximum pour les solutions d'un système fortement elliptique, Glasnik Mat. Ser. III 4 (24) (1969), 75-78. MR 39 \#1792.

DEPARTMENT OF MATHEMATICS, STANFORD UNIVERSITY, STANFORD, CALIFORNIA 94305 Israel

Current address: Institute of Mathematics, The Hebrew University, Jerusalem, 